\title{
DeephageTP: A Convolutional Neural Network Framework for Identifying Phage-specific Proteins from metagenomic sequencing data
}

\section{Yunmeng Chu}

Shenzhen Institutes of Advanced Technology Chinese Academy of Sciences

\section{Shun Guo}

Shenzhen Institutes of Advanced Technology Chinese Academy of Sciences

Dachao Cui

Shenzhen Institutes of Advanced Technology Chinese Academy of Sciences

Haoran Zhang

Shenzhen Institutes of Advanced Technology Chinese Academy of Sciences

xiongfei Fu

Shenzhen Institutes of Advanced Technology Chinese Academy of Sciences

Yingfei Ma ( $\nabla$ yingfei.ma@siat.ac.cn )

Shenzhen Institutes of Advanced Technology https://orcid.org/0000-0002-2563-5390

\section{Methodology}

Keywords: Convolutional Neural Network(CNN), deep learning, phage, metagennomics, phage-52 specific protein

Posted Date: April 21st, 2020

DOl: https://doi.org/10.21203/rs.3.rs-21641/v1

License: (c) (i) This work is licensed under a Creative Commons Attribution 4.0 International License. Read Full License 
1 DeephageTP: A Convolutional Neural Network Framework for Identifying

2 Phage-specific Proteins from metagenomic sequencing data

3 Running title: an alignment-free deep learning framework for identifying phage-

$4 \quad$ specific proteins

$$
\begin{aligned}
& \text { Yunmeng Chu }{ }^{1,2,3,4 \#} \text {, ym.chu@siat.ac.cn } \\
& \text { Shun Guo }{ }^{1,2,3 \#} \text {, shun.guo@siat.ac.cn } \\
& \text { Dachao Cui }{ }^{1,2,3 \#} \text {, dc.cui@siat.ac.cn } \\
& \text { Haoran Zhang }{ }^{1,2,3} \text {, hr.zhang@siat.ac.cn } \\
& \text { Xiongfei Fu }{ }^{1,2,3} \text {, xf.fu@siat.ac.cn } \\
& \text { Yingfei Ma }{ }^{1,2,3^{*}}, \text { yingfei.ma@siat.ac.cn }
\end{aligned}
$$

2Shenzhen Key Laboratory of Synthetic Genomics, Shenzhen, 518055, China.

${ }^{3}$ Guangdong Provincial Key Laboratory of Synthetic Genomics, Shenzhen, 518055, China.

${ }^{4}$ Department of Bioengineering and Biotechnology, Huaqiao University, Xiamen, Fujian, 361021, PR China.

\#These authors contributed equally.

*Corresponding to Yingfei Ma, yingfei.ma@siat.ac.cn 


\section{Abstract:}

Background: Bacteriophage (phage) is the most abundant and diverse biological entity on the Earth. This makes it a challenge to identify and annotate phage genomes efficiently on a large scale. Portal (portal protein), TerL (large terminase subunit protein) and TerS (small terminase subunit protein) are the three specific proteins of the tailed phage. Here, we develop a CNN (convolutional neural network)-based framework, DeephageTP, to identify these three proteins from metagenome data. The framework takes one-hot encoding data of the original protein sequences as the input and extracts the predictive features in the process of modeling. The cutoff loss value for each protein category was determined by exploiting the distributions of the loss values of the sequences within the same category. Finally, we tested the efficacy of the framework using three real metagenomic datasets.

Result: The proposed multiclass classification CNN-based model was trained by the training datasets and shows relatively high prediction performance ( Accuracy: Portal, 98.8\%; TerL, 98.6\%; TerS, 97.8\%) for the three protein categories, respectively. The experiments using the independent mimic dataset demonstrate that the performance of the model could become worse along with the increase of the data size. To address this issue, we determined and set the cutoff loss values (i.e., TerL: -5.2 , Portal: -4.2 , TerS: -2.9) for each of the three categories, respectively. With these values, the model obtains high performance in terms of Precision in identifying the TerL and Portal sequences (i.e, 94\% and $\sim 90 \%$, respectively) from the mimic dataset that is 20 times larger than the training dataset. More interestingly, the framework identified from the three real metagenomic datasets many novel phage sequences that are not detectable by the two alignment-based methods (i.e., DIAMOND and HMMER).

Conclusions: Compared to the conventional alignment-based methods, our proposed framework shows high performance in identifying phage-specific protein sequences with a particular advantage in identifying the novel protein sequences with remote homology to their known counterparts in public databases. Indeed, our method could also be applied for identifying the other protein sequences with the characteristic of high complexity and low conservation. The DeephageTP is available at https://github.com/chuym726/DeephageTP.

Keywords: Convolutional Neural Network(CNN), deep learning, phage, metagennomics, phagespecific protein 


\section{Background}

Bacteriophages (phages) are the most abundant and diverse biological entities on the Earth. With the advent of the high-throughput sequencing technologies, the amount of microbial metagenomic sequencing data is growing by exponential order. Phages are widely present in various environments and thus the phage-originated sequences are present in the microbial metagenomic sequencing data. Particularly, it is estimated that around $17 \%$ sequences of the human gut metagenomes are derived from phage genomes [1]. However, it remains a challenge to identify phage-derived sequences from the metagenomic sequencing data due to the following aspects: (a) the phage genomes are highly diverse and lack of universal marker genes akin to 16S rRNA genes of bacteria or archaea [2]; (b) most of the phages are uncultured as their parasitism relies primarily on the host bacteria [3]. These limit our investigations into the complex microbiota for the understanding of the roles of the phages in the complex ecosystems.

To identify the phage-derived sequences from the complex microbial metagenomic sequencing data, one common practice is to examine the phage-specific genes carried by the metagenomic sequences. Thus, if a given predicted protein sequence shows significantly high similarity with the specific proteins of known phages, the metagenomic sequence carrying the protein could be selected as the candidate of the phage-derived sequence. In this regard, several alignment-based methods have been developed and extensively utilized, such as BLAST, PSI-BLAST [4], HMM (Hidden Markov Models) [5], etc. Nonetheless, these alignment-based methods mainly rely on reference phage sequences, usually leading to the failure of detecting the novel phages that encode proteins with poor similarity to those of the reference phages.

Recently, many alignment-free algorithms have been developed for identifying and annotating the proteins. Specifically, they typically convert each sequence into a feature vector and then, the computational prediction of the sequence is implemented based on the corresponding feature vector. For instance, several machine learning-based methods [6-13] utilize the amino acid frequency as the main predictive features of the sequences to identify phage-specific proteins, and the representative methods include VIRALpro [10], PVP-SVM [11], iVIREONS [6], etc. One main problem of these methods is that, the possible combinations of amino acids (i.e., $20^{k}, k$ is the length of amino acid fragments) are too many. This makes the dimension of the feature vector difficult to tolerate the increase of $k$. Therefore, these methods usually set the value of $k$ less than 4 . This, in turn, will lead 
to the loss of the information, and thus, the prediction performance of the algorithms could be significantly impaired [14]. Among alignment-free methods, some deep-learning based models show promising performance, such as DeepFam [14], DEEPre [15], mlDEEPre [16], DeepFunc [17], DeepGo [18], etc. Most recently, DeepCapTail [19] has been proposed for predicting capsid and tail proteins of the phage using deep neural network. It suffers from the same limitation of utilizing the amino acid frequency as the predictive features of the sequences. Moreover, it has not been applied to the real metagenomic dataset for examining the actual effect.

To overcome these limitations, in this study, we develop a framework DeephageTP (Deep learning-based phage Terminase and Portal proteins identification) for identifying the three tailedphage-specific proteins, i.e., TerL (large terminase subunit), Portal and TerS (small terminase subunit). Especially, this framework proposes Convolutional Neural Network (CNN)-based deep learning model that is allowed to take the original sequences as the input and extract the corresponding predictive features from the sequences in the process of modeling. Moreover, we present a new strategy to tackle the false positives problem along with the increasing size of the testing datasets by setting the cutoff value for each category of interest. Finally, the proposed framework was applied on three real metagenomic datasets, and the results indicate that our method would be an effective complement of the mainstream alignment-based methods to identify the phage-specific functional proteins with relatively high accuracy. Thus, our proposed framework provides the potential opportunity to recognize the new phage at a large scale from metagenomic datasets.

\section{Materials and Methods}

\section{Datasets}

The initial collection of phage protein sequences was obtained from the database: Uniport (www.uniprot.org). The molecular machine of the tailed phage is typically comprised of three proteins, i.e., portal (Portal protein), motor (large terminase subunit protein, TerL) and regulator (small terminase subunit protein, TerS). Because these proteins are crucial in packaging the phage genome $[20,21]$, thus the metagenomic sequences carrying the genes of these proteins can be identified as the phage sequences. Without loss of generality, we focus on these proteins in this study. The steps of constructing the training dataset are described as follows (Fig. 1A): i) according to the taxonomy in the UniProt database, all proteins in archaea, bacteria and viruses were obtained from the database; ii) the protein sequences were searched by the keywords (i.e., portal, large terminase subunit, and 
small terminase subunit), and the noise sequences with the uncertain keywords (e.g., hypothetical, possible, like, predicted) were removed to ensure that the selected protein sequences in the three categories are veracious; iii) the remaining sequences without the keywords of interest (portal, large terminase subunit and, small terminase subunit) were labeled as the 'others' category. However, the size of the 'others' category is more than 75 times larger than that of the three categories. To relieve the class-imbalance problem brought by this situation, we randomly selected 20000 protein sequences from the remaining sequences and labeled as the 'others' category; iv) to further guarantee that the sequences from the database with the three categories are veracious, we calculated length distribution of these sequences (see Fig. S1), then manually checked the sequences with the abnormal length $(<$ 5\% and $>95 \%$ ) using Blastp (https://blast.ncbi.nlm.nih.gov/Blast.cgi) against NCBI nr database, and the sequences without hitting to the targeted references were filtered out (almost all the sequences with abnormal length) and labeled as the 'others' category. The training dataset is summarized in Table 1.

To test the proposed model, we also constructed a mimic metagenomic dataset by collecting the protein sequences from another database: UniRef100 (https://www.uniprot.org/uniref/). The collection process for the mimic metagenomic dataset is similar to that of the training dataset. It should be noted that the two databases (i.e., UNIPROT and UniRef100) have some overlaps, and thus we manually deleted the sequences that exist in the training dataset from the mimic dataset. To this end, the mimic dataset can be regarded as an independent dataset from the training dataset. Particularly, to investigate the prediction performance of the model on the test data with different size, we generated 7 groups of data (i.e., Group 1 Group 7) from the original mimic dataset (i.e., Group 8), where except for the three category proteins, the samples from the 'others' category were randomly selected from the Group 8. Here, since we mainly focus on the impact of different data sizes on the performance of the proposed model in identifying the three category proteins, the samples of the three category proteins were kept the same for 8 groups of the data. Table 2 describes the details of the datasets used for testing analysis.

To verify the performance of the proposed model on the real metagenomic dataset, we collected the virome dataset from the wastewater (accession number in NCBI: SRR5192446) and two virome datasets from the human gut (accession number in NCBI: SRR7892426 and ERR2868024) [22, 23]. As the data of these datasets are raw reads, we first assembled them using SPAdes 3.11.1 [24] and applied Prodigal [25] for gene calling with the default parameters. As a result, we obtained 366146 
(SRR5192446), 110129 (SRR7892426) and 27157 (ERR2868024) protein sequences for these datasets, respectively.

\section{Protein sequence encoding}

To tackle the protein sequence data with the proposed model, we firstly formulated an image-like scheme to encode each protein sequence (Fig. 1B). Specifically, each of the 20 amino acids is encoded as a one-hot vector of 20 dimensions (i.e., one-dimension value is 1 and others are 0 , shown in Fig. 1B) [26]. Based on this, a protein sequence with $L$ length (i.e., the number of amino acid residues) could be encoded as a $L \times 20$ matrix $X$.

As the lengths of the protein sequences typically varied, while the input data are required to be the same size for the model, we fixed len_w (the maximum length of the sequence for modeling) equal to 900 according to the length distribution of the three category proteins (almost all lengths of the three proteins are less than 900). Specifically, if the length of a given sequence is longer than len_w, the excess part of the sequence would be abandoned; else, the insufficient part of the sequence would be filled with multiple '-'. Each '-' is encoded as a zero vector of 20 dimensions. In the light of this, each protein sequence could be encoded as a $l e n_{-} w \times 20$ matrix. These matrixes can be used as the input data for the proposed model.

\section{CNN-based deep learning model}

The framework DeephageTP is developed based on the algorithm of CNN. The CNN comprises a convolutional layer, a max-pooling layer, two fully connected layers as well as the input and output layers. The dropout technique [27], which avoids overfitting via randomly removing the units at some rates (i.e., 0.1 in our experiments), is applied on the pooling layer and the first fully connected layer in the model. One of the most common activation function ReLu [26] is used on the convolutional layer and the first connected layer, while the output layer utilizes SoftMax [28] as the activation function to compute the probability of the protein sequence against the category. The CNN model is shown in Fig. 1C.

It is worth noting that there are many hyperparameters in the model such as the number of the convolution kernels, the number of units in fully connected layers, the dropout rate, the learning rate, etc. However, it is difficult to obtain the optimal values of these parameters. To this end, for most of these parameters, in the process of modeling, we used the default settings that are widely applied in practice [26], while the remaining parameters were tuned according to the averaged prediction 
performance of the proposed model on the training dataset using the 5-fold cross-validation. The structure of the CNN was determined by examining four main hyper-parameters [29], including the length size of protein sequence, kernel size of the filter, number of filters for each kernel size and the number of neurons in fully connected layer [14]. These parameters were selected according to our experiences and the references [30,31]. The protein sequence of 20 amino acids were classified into 7 groups (7-letter reduced sequence alphabets) according to their dipole moments and side-chain volume: $\{\mathrm{A}, \mathrm{G}, \mathrm{V}\},\{\mathrm{I}, \mathrm{L}, \mathrm{F}, \mathrm{P}\},\{\mathrm{Y}, \mathrm{M}, \mathrm{T}, \mathrm{S}\},\{\mathrm{H}, \mathrm{N}, \mathrm{Q}, \mathrm{W}\},\{\mathrm{R}, \mathrm{K}\},\{\mathrm{D}, \mathrm{E}\}$ and $\{\mathrm{C}\}[32]$. The kernel size of the filter was set to $7 \times 1$ in the light of the previous studies [32,33]; we examined the values of 800 , 900 and 1000 for the length of sequences based on the distribution of the length; we also examined the values of 30, 50, 70 and 90 for the number of filters, as well as the values of 50, 100, 150 and 200 for the number of neurons in the fully connected layer. Specifically, we evaluated the performance of the model with different values of the parameters using 5-fold cross-validation on the training dataset, and the results are shown in Table S1-3. Finally, we set the length size to 900, the number of filters to 50 , and the number of the neurons in the fully connected layer to 100.

The architecture of the DeephageTP framework is implemented using the Python Keras package (https://keras.io), a widely applied, highly modular deep learning library. The DeephageTP is available at https://github.com/chuym726/DeephageTP.

In summary, as shown in Fig. 1A, in this study, the proposed DeephageTP framework was firstly implemented on the training dataset for feasibility analysis, and then the trained model was applied on the mimic dataset for test and the cutoff value of each category of interest was determined according to the responding loss values distributions; finally, we applied the trained model on the real metagenomic datasets for examining the performance of our framework.

\section{Evaluation metrics}

To evaluate the performance of the proposed model, four widely used metrics, i.e., Accuracy, Precision, Recall, and F1-score were applied in this study and defined as:

$$
\text { Accuracy }=\frac{T P+T N}{T P+F P+T N+F N},
$$

$$
\text { Precision }=\frac{T P}{T P+F P},
$$

$$
\text { Recall }=\frac{T P}{T P+F N},
$$


where TP denotes true positives (i.e., a protein sequence from one of the categories is predicted correctly as the category), TN (true negatives, a protein sequence comes from other categories of interest is predicted correctly as the other category), FN (false negatives, a protein sequence comes from the category of interest is wrongly predicted as the other category), and FP (false positives, a protein sequence comes from a different category is wrongly predicted as the category of interest). Accuracy reflects the overall prediction quality of the model. Precision focuses on measuring how accurate the categories of the phage protein sequences predicted by the model are, while Recall measures the proportions of the phage protein sequences that are correctly identified by the model. And F1-score is the harmonic mean of Precision and Recall.

\section{Loss value computation}

To determine the appropriate cutoff loss values for the three protein categories, we considered the loss value of each sequence. The loss value is calculated according to the loss function used in the proposed model. The loss value is a score criterion that reflects the difference between the real category of the sequence and the predicted category of the sequence. The smaller the loss value is, the smaller the difference is. Specifically, the widely applied cross-entropy function [26] was employed in this study and defined as follows:

$$
L=-\sum_{k=0}^{K-1} y_{k} \log p_{k}
$$

where $y_{k}$ is the value of the real label of the sequence on the $k$-th dimension, and $p_{k}$ is the corresponding value on the $k$-th dimension that predicted by the model. For most deep learning models, the category label is typically encoded as a one-hot vector (i.e., one-dimension value is 1 and others are 0 ) with $k$ dimensions, and the predicted value for each dimension is calculated via the SoftMax function.

Additionally, in general, the averaged loss value for all sequences is applied for evaluating the performance that the model fits the dataset. However, in this study, we utilized the loss value for each sequence to determine the cutoff values. The main reason is that, if a sequence is predicted as one category by the trained model with a very small loss value, it means that the sequence is much the same as the sequences within the category, and the smaller the value is, the more likely it would be. On the other hand, if the loss value is relatively large, although the sequence is predicted as the 
category by the model, it would likely be false positives. To this end, according to the distribution of the loss values with the same category, the bounds that distinguishing TP and FP will be determined.

\section{DeephageTP application on real metagenomic datasets}

To assess the performance of the proposed framework on real metagenomic data in identifying the phage-derived sequences, we applied the framework on the three real metagenomic datasets. Specifically, the protein sequences of the three categories predicted by the framework were selected and then filtered with the cutoff loss values. Finally, we manually checked the DeephageTP-identified protein sequences using Blastp (cutoff e-value=1e-10) against the NCBI nr database. According to the results, the identified sequences can be divided into four groups: a) true-positive: the sequence has Blastp hits in the NCBI nr database within the same category as DeephageTP predicted (as long as one hit in the result list of Blastp against NCBI nr database is annotated to the category of interest); b) phage-related: at least one of the protein sequences carried by the contig where the identified protein gene is located has hits to other phage-related proteins (as long as one is annotated to phagerelated protein in the result list of Blastp); c)Unknown, the sequences don't have hits or the hits are annotated as hypothetical protein; d)Other function, the sequences have hits annotated as other functional proteins that likely are derived from bacterial genomes (none of the hits in the result list of Blastp are annotated as phage-related proteins).

\section{Alignment-based methods for comparison}

Two major alignment-based methods, Hidden Markov Model (HMM) [34] and Basic Local Alignment Search Tool (BLAST) [4] were used to annotate the protein sequences and the results were compared with those of our method in the experiment. Specifically, multiple sequence alignments were generated firstly using MUSCLE v3.8 [35] for the categories of interest in the training dataset. Then, the HMM algorithm was constructed using HMMER v3.1(http://hmmer.org/). For each sequence alignment, we built HMM of each protein category via hmmbuild, where the models were compressed into a single database indexed with hmmpress. For each test protein sequence, hmmscan scored the significance that the sequence matched to the categories of interest with E-value, and the category with the most probable (i.e., the one with the smallest E-value) was chosen as the output. In some cases, the E-value could not be yielded from the constructed models, where the sequences were discarded in our experiment. The two software (i.e., MUSCLE v3.8 and HMMER v3.1) were set with default parameters for implementation. For the BLAST method, we used the software DIAMOND 
[36] to find the most similar sequence in the database (created with the proteins in our training dataset) for a test protein sequence and assign its category to the test sequence. The cutoff e-value of the DIAMOND program was set 1E-10 in our experiment.

\section{Results}

\section{Prediction performance of the CNN-based model on the training dataset}

In the training dataset, $80 \%$ sequences of each category were randomly selected for training the proposed model, while the remaining 20\% were used for the test. The results are shown in Fig. 2A. As it can be observed that, in general, the proposed model show relatively high prediction performance on the dataset; over $\mathbf{9 7 \%}$ accuracy can be achieved for the three protein categories obtained on the protein Portal in terms of Precision, Recall and F1-score $(93.88 \%, 96.94 \%$, and 95.33\%, respectively). The relatively high prediction performance achieved for TerL (Precision: 93.75\%, Recall: $91.60 \%$, F1-score: $92.66 \%$, respectively). The prediction of TerS generated the lowest performance (Precision: 75.28\%, Recall: 91.03\%, F1-score: 82.41\%, respectively), especially for Precision, suggesting that nearly a quarter of TerS sequences could not be correctly identified by the model.

\section{Prediction performance of the $\mathrm{CNN}$-based model on the mimic metagenomic dataset}

To further assess the proposed model, we prepared an independent mimic metagenomic dataset from another database: UniRef100. We applied the trained model on the mimic dataset (Group 8) (Table 2). As shown in Fig. 2B, we found that, except for Accuracy, the prediction performances in terms of other metrics significantly became worse (TerL 71.1\%, Portal 70.5\%, TerS 19.1\% (Precision); TerL 82.3\%, Portal 73.0\%, TerS 73.9\% (Recall); TerL 76.3\%, Portal 71.7\%, TerS 30.3\% (F1-score)) for the three proteins when compared with those on the training dataset. This is likely because, in the mimic dataset, the number of the sequences from the 'others' category is much larger than that of the sequences from the category of interest (i.e., class imbalance).

Thus, we further applied the trained model on the seven groups of the data, respectively, to assess the impact of such class imbalance on the prediction performance of the model in identifying the three phage-specific protein sequences. The mimic dataset was divided into 7 groups with different sizes (Table 2). The results are shown in Fig. 3 and Table S4. Compared with the results on Group1, 
Precision and F1-score values for the three proteins decreased significantly (by TerL 1.6\% 23.2\%, Portal 1.5\% 26.4\%, TerS 7.0\% 49.5\% (Precision); TerL 0.7\% 11.6\%, Portal $0.6 \% \sim 11.6 \%$, TerS $15.6 \% \sim 52.4 \%$ (F1-score) ) with the dataset size increasing, while the Recall values remain unchanged. This indicates that the number of true-positive sequences from the categories of interest was not impacted by the size of the dataset. However, with the testing dataset size increasing (Table2), more and more sequences from the 'others' category were wrongly predicted as the category of interest by the model (i.e., the FP value becomes larger). Since the Recall values are the same for all testing datasets, the F1-score values are only affected by the Precision values and the trend of the F1-score values are similar to that of the Precision values. Therefore, we focus on the prediction performance in terms of Precision in the following experiments.

Therefore, we further employed a new strategy to improve the prediction performance of the model in terms of Precision by introducing the appropriate cutoff loss value for each category of interest. Specifically, we first calculated the distributions of the loss values of the sequences correctly identified (i.e., TP) and the sequences wrongly predicted as the categories of interest (i.e., FP) by the trained model for the three protein categories using the 8 groups of the mimic metagenomic dataset, respectively (Table 2); based on this, the loss value for a given category that may distinguish the TP and NP for most sequences would be chosen as the corresponding cutoff value. It should be noted that, as mentioned above, the TP values of the three protein categories are the same in the 8 groups of the mimic metagenomic datasets, so are the distributions of the corresponding loss values. As shown in Fig. 4, since the majority of the loss values of TP sequences are relatively low (loss values: TerL $<-5.2$, Portal $<-4.2$, TerS $<-2.9$ ) while those of FP sequences are relatively high (loss values: TerL $>-4.0$, Portal $>-3.6$, TerS $>-2.5$ ) for the three proteins on all groups, thus, the corresponding cutoff values of three phage proteins for distinguishing TP and FP could be selected with relative ease. Because the distributions of the loss values for three proteins are different, thus it is essential to set the appropriate cutoff values for each of them. In this study, we chose the values at the top of the boxplots of the three TP protein sequences in Fig. 5 (i.e., TerL: -5.2, Portal: -4.2, TerS: -2.9) as the cutoff values for the three categories, respectively. With these cutoff values, we can observe most TP sequences ( $>99 \%$ ) in the mimic metagenomic dataset (group 8) were identified correctly. Clearly, a stricter cutoff value could also be selected according to the practical necessity and the consideration of the balance between false positive rate and false negative rate. 
With the determined cutoff loss values, we reassessed the prediction performance of the model on the 8 groups of the mimic metagenomic dataset. Specifically, the sequences that originally were predicted as the category of interest but with the loss value larger than the corresponding cutoff value would be predicted as the 'others' category instead. As shown in Fig. 3, Table S4 and Table S5, compared with the results obtained without using the cutoff values, the performance of the new strategy shows remarkable improvements in terms of Precision (improved by TerL 4.9 22.8\%, Portal 2.2 19.3\%, TerS $22.2 \sim 43.5 \%$ ) for the 8 groups, although the prediction performance in terms of Recall somewhat decreases. Moreover, compared to the result of group 1, with the increasing sizes of the groups, the Precision values reduced by TerL $0.3 \sim 5.3 \%$, Portal $0.5 \sim 9.4 \%$, TerS $1.5 \sim 28.1 \%$ for the three proteins, which were much less than those of without using the cutoff strategy. In particular, the Precision values for TerL and Portal can still reach $\sim 94 \%$ and $\sim 90 \%$ respectively, even on the that, by introducing the cutoff values, the effect of the excessive size of the testing data would be reduced to a relatively small degree.

It worth noting that, in all these experiments, the model showed much worse prediction performance in identifying TerS sequences than the other two proteins (Fig. 3, Table S4, S5), although the introduction of cutoff loss value can significantly improve the performance of the model in terms of Precision(21 42\%). This is likely because the number of TerS used for training is much less than those of the other two proteins.

\section{Application of the framework DeephageTP on the real metagenomic datasets}

We applied the framework on the three real metagenomic sequencing datasets with the corresponding cutoff loss values $((\log 10)$ : TerL: -5.2 , Portal: -4.2 , TerS: -2.9$)$ to identify the phagederived sequences. Finally, 1185 out of 366146 protein sequences (TerL: 147, Portal: 341, TerS: 697) were identified from the dataset (SRR5192446) by our method, 42 out of 27157 protein sequences (TerL: 9, Portal: 15, TerS: 18) from ERR2868024 and 127 out of 110129 protein sequences (TerL: 16, Portal: 23, TerS: 88) from SRR7892426. The dataset (SRR5192446) has a higher number of identified sequences of interest than the other two. This result is in line with those of two alignment-based methods (i.e., DIAMOND and HMMER). It can be observed that the total numbers of the three phage proteins predicted from the sample (SRR5192446) by the two alignment-based methods are 4200 (DIAMOND) and 357 (HMMER) respectively, much higher than those from the other two datasets (ERR2868024, and SRR7892426). This is likely because the sample (SRR5192446) was collected 
from the environment of waste-water and the majority of the sequences in the training dataset were collected using environmental microbes. Among the protein sequences identified by the three methods from the dataset of waste-water (SRR5192446), quite a few sequences (TerL 85, Portal 105, TerS 13) are shared by DeephageTP, and DIAMOND, some (TerL 9, Portal 3, TerS 0) shared by DeephageTP and HMMER, but very few can be identified by the three methods simultaneously (Fig. 5), suggesting that the phage-specific protein sequences identified by DeephageTP are different from those of alignment-base methods, and these protein sequences are likely derived from novel phage genomes in the metagenomes. This case is similar to those of the other two datasets from human gut samples(Fig. S2).

To further confirm the sequences identified by DeephageTP, we manually checked the protein sequences using Blastp (E-value:1e-10) against the NCBI nr database. As shown in Fig. 6, the results demonstrate that, again, few DeephageTP-identified TerS sequences were verified in NCBI nr database as true positive (SRR5192446: 22 (3.16\%), ERR2868024: 1 (5.56\%), SRR7892426: 4 $(4.55 \%))$. However, in regard to TerL and Portal, a large fragment of the protein sequences were confirmed as the true positive (SRR5192446: TerL 105 (71.4\%), Portal 172 (50.4\%); ERR2868024: TerL 5 (55.6\%), Portal 7 (46.7\%); SRR7892426: TerL 12 (75\%), Portal 16 (69.6\%)).We further examined the whole contigs that carry the remaining identified protein sequences. According to the hits of each protein carried by the contigs, only a small number of identified proteins belong to other functional proteins likely encoded by bacterial genomes (SRR5192446: TerL 6 (4.1\%), Portal 7 (2.1\%); ERR2868024: TerL 0 (0\%), Portal 1 (6.7\%); SRR7892426: TerL 0 (0\%), Portal 0 (0\%)). Note that, a considerable proportion of the identified proteins are encoded by phage-derived contigs (SRR5192446: TerL 20 (13.6\%) Portal 103 (30.2\%) TerS 243 (34.9\%), ERR2868024: TerL 3 (33.3\%) Portal 6 (40\%) TerS 8 (44.4\%), SRR7892426: TerL 4 (25\%) Portal 5 (21.7\%) TerS 31 $(35.2 \%))$ and quite a part of the predicted proteins belong to unknown proteins (SRR5192446: TerL 16 (10.9\%), Portal 59 (17.3\%) TerS 351 (50.4\%), ERR2868024: TerL 1 (11.1\%) Portal 1 (6.7\%) TerS 0 (0\%), SRR7892426: TerL 0 (18.75\%) Portal $2(8.7 \%)$ TerS $22(25 \%))$. Most of these proteins have low identities $(<30 \%)$ (Table S6) to the hits in the NCBI nr database, suggesting some of them are likely novel TerL encoded by novel phages, which needs further investigations. Among the protein sequences identified by DeephageTP and confirmed as the true positive, a number of proteins were not determined by the other two alignment-based methods (Table S6). For example, 10.2\%(15/147) TerLs and 37.8\%(65/172) Portals were only detected by DeephageTP in sample SRR5192446. This 
indicates that DeephageTP is capable of recognizing novel phage genes of interest. These novel genes are great divergent from their reference ones, and thus, may be ignored by alignment-based methods.

\section{Discussion}

Bacteriophages are present in all kinds of the microbial microbiome. With conventional sequence-alignment-based methods, the identification of phage sequences from the metagenomic sequencing data remains a challenge due to the great diversity of phages and the lack of conserved marker genes among all phages. In this paper, we present a CNN-based deep learning framework, DeephageTP, an alignment-free method to identify three tailed-phage-specific proteins, i.e., TerL, Portal, and TerS. In doing so, we can further recognize phage-derived sequences carrying the three proteins from metagenome sequencing data. The CNN-based model is trained by inputting the specific features extracted from one-hot vectors of 20 dimensions encoded by the protein sequences, and thus can efficiently identify the hidden patterns that are difficult to be detected by other bioinformatics techniques. With the introduction of cut-off loss values, the performance of the framework can be significantly improved in terms of Precision. More importantly, compared with the two alignment-based methods, the proposed framework in this study has the advantage of identifying novel phage sequences from real metagenomic sequencing data.

We employed the multiclass classification CNN model in this study. In general, the identification of the three proteins can be deemed as three binary classification problems (one-vs-all scheme) or a multiclass classification problem. The former divides the original data into two-class subsets and learns a different binary model for each new subset. It may bring more cost of calculation than the latter as it learns multiple different models. We also compared the prediction performances of these two strategies using the training dataset, and the results are shown in Table 3. It can be seen that the two strategies have similar prediction performance to a large extent. Specifically, for TerL, the binary models performed a bit better than the multiclass model (Accuracy: 98.82\% vs 98.58\%; Precision: 95.45\% vs 93.75\%; Recall: $91.98 \%$ vs 91.60\%; F1: 93.68\% vs 92.67\%). For Portal, the binary models achieved better performance in terms of Accuracy, Precision and F1 (Accuracy: 99.24\% vs 98.84\%; Precision: $99.19 \%$ vs 93.78\%; F1: 96.7\% vs 95.33\%). Meanwhile, the multiclass model obtained better prediction performance in terms of Accuracy, Precision and F1 (Accuracy: 97.83\%vs 96.96\%; Precision: $75.28 \%$ vs $65.80 \% ; F 1: 82.41 \%$ vs $76.73 \%$ ) for TerS. Considering the cost of computation, we used the multiclass classification model rather than the binary classification models in this study. 
In microbial metagenomic sequencing datasets, only a small fragment of sequences is derived from the phage genomes. This class imbalance problem can affect the performance of our framework. We applied the trained model on an independent mimic metagenomic dataset (20 times larger than the training dataset) and found that the prediction performance in terms of Precision, Recall, and F1score decreased remarkably. In the mimic dataset, many sequences from the 'others' category are different from those in the training dataset, and these sequences are wrongly identified as the category of interest by the trained model (i.e., false positive problem). This likely leads to the reduction of Precision. Meanwhile, a part of sequences belong to the category of interest would be dissimilar to those in the training dataset; thus, they are wrongly predicted as the other category by the trained model (i.e., false negative problem), where it results in the reduction of Recall. Clearly, the descent degree of Recall is less than that of Precision, especially for TerS. The reduction of F1-score is inevitable as it is the harmonic mean of Precision and Recall.

To further examine the impact of the data size on the prediction performance of the model, we conducted the experiments on the 7 additional groups from the mimic metagenomic dataset with different sizes. An interesting finding was that, for the 8 groups, the prediction performance in terms of Recall was not affected by the data size, while the prediction performance in terms of Precision decrease significantly with the increase of the data size. Here, we presented a new way to improve the prediction performance of the proposed model in terms of Precision by introducing the cutoff loss values that were determined according to the distribution of the loss values with the category of interest. This strategy can significantly improve the prediction performance of the model in terms of Precision for the categories of interest. The larger the size of the testing dataset is, the more significant the improvement of the performance will be. On the other hand, the prediction performance in terms of Recall was reduced unavoidably with the strategy compared to the results without the strategy, which means the false negative rate was raised. Even so, our strategy provides a certain basis for setting a cutoff value of each category that will balance the FP rate and the FN rate.

Our framework demonstrated a remarkable capability to identify new phage protein sequences that have extremely low identities with the known sequences of the training data. In the testing analysis, the framework identified the majority of the three protein sequences (Recall, 82.3\% TerL, 73.0\% Partal and 74.0\% TerS, Fig. 2B, Table S4) from the mimic metagenomic dataset where all the three protein sequences are different from those of the training dataset. Moreover, in the application of the framework on the real metagenomic datasets, the capability of the framework in identifying 
novel phages also can be observed that our method identified many phage protein sequences that were missed by the two alignment-based methods. In this study, we verified the novelty of the DeephageTP-identified sequences by reannotating them in the NCBI nr database. Experiments including gene express and Transmission Electron Microscope, which are the gold standard for identifying phage particles, are required in further studies.

Nonetheless, we also observed some limitations of the proposed framework in the application. First, it seems that only a small number of the phage sequences present in the metagenomic data can be identified by the proposed framework. For example, in sample SRR5192446, 147 (106 true positive) TerL sequences and 341(172 true positive) Portal sequences were identified, as compared with 2581 and 1295 by the software DIAMOND, respectively. Similar cases were also observed in the other two human gut samples (Fig. S2). This proportion of the identified sequences against the phage sequences that are estimated to be present in the virome datasets is relatively low [22, 23]. Also, the framework failed to identify the crAssphage-like phages which are known widely distributed in human gut samples (Table S6). Second, our trained model likely prefers to identify the phages of the environmental microbes instead of those of the human gut microbes. Around $0.029 \%(106 / 366146)$ of the sequences were identified as true positive TerL sequences by the framework from the water sample, while only $0.018 \%(5 / 27157)$ and $0.011 \%(12 / 110129)$ from the other two human gut samples, respectively. This is likely because the phage sequences recruited by the training dataset are mainly from environmental samples, and in the NCBI nr database, more than $98 \%$ phages are specific to infect the environmental microbes. Third, the performance of the proposed framework in identifying TerS sequences from metagenomic datasets is relatively low in contrast to TerL and Portal sequences. In general, in a given metagenome, the number of TerS is equal to that of TerL, but in all cases in our study, the number of TerSs identified by the framework is around one-fifth of that of TerLs. All these limitations of the proposed framework can be attributed to the extremely small number (TerL 2617, Portal 3260, TerS 1503) of the known phage sequences included in the training dataset, compared to the number of phages present in the environmental samples and human gut samples. Therefore, the information extracted from the limited number of known phages using the framework is insufficient to cover all phage sequences in a given metagenomic sample. Particularly, the low performance of the framework in identifying TerS sequences might be because the number of TerS sequences used for training is much less and the length of the sequences is shorter than those of other two proteins, and the information provided by the TerS sequences in training dataset would be insufficient to 
identify the different TerS sequences in the metagenomic datasets. The shorter the sequence is, the less information is provided to the framework. Thus, to optimize our proposed framework in further study, we will select the appropriate marker sequences with a longer length and include more sequences into the training dataset.

\section{Conclusions}

In summary, we devise and optimize a CNN-based deep learning framework for identifying the three phage-specific protein sequences from complex metagenomic sequencing datasets. Compared to the conventional alignment-based methods, our proposed framework shows a particular advantage in identifying the novel protein sequences with remote homology to their known counterparts in public databases. Indeed, our method could also be applied for identifying the other protein sequences with the characteristic of high complexity and low conservation, where it would be another interesting way to explore.

\section{List of abbreviations}

TerL (large terminase subunit protein)

TerS (small terminase subunit protein)

CNN (convolutional neural network

DeephageTP (Deep learning-based phage Terminase and Portal proteins identification)

\section{DECLARATIONS}

\section{Ethics approval and consent to participate}

Not applicable

\section{Consent for publication}

Not applicable

\section{Availability of data and material}

The python code of DeephageTP is available at https://github.com/chuym726/DeephageTP. All data needed to evaluate the conclusions in the paper are present in the paper and/or the Supplementary Materials. Additional data related to this paper may be requested from the authors.

\section{Competing interests}

The authors declare that they have no competing interests. 


\section{Funding}

This study was supported by the Ministry of Science and Technology of China (http://www.most. gov.cn, grant nos. 2018YFA0903100). This study was also supported by the grant from Guangdong Provincial Key Laboratory of Synthetic Genomics (2019B030301006), Shenzhen Key Laboratory of Synthetic Genomics (ZDSYS201802061806209), and the Shenzhen Peacock Team Project (KQTD2016112915000294).

\section{Authors' contributions}

Y.M and S.G designed the research. Y.C, S.G, D.C and, H.Z performed analysis. S.G., Y.M., and Y.C drafted the paper. All authors contributed to the interpretation of the results and to the text.

\section{Acknowledgements}

Not applicable

\section{Supplementary Materials}

Fig. S1. The length distribution of the three protein sequences.

Fig. S2. The Venn diagrams of the prediction results of three methods (i.e., DeephageTP, Diamond and HMMER) on the metagenomic datasets. ERR2868024: A(TerL), B(Portal), C(TerS); SRR7892426: D(TerL), E(Portal), F(TerS).

Table S1. The average loss value and the average accuracy of 5-fold cross-validation on the training dataset with different sequence length sizes.

Table S2. The average loss value and the average accuracy of 5-fold cross-validation on the training dataset with the different number of filters.

Table S3. The average loss value and the average accuracy of 5-fold cross-validation on the training dataset with the different number of neurons in the fully connected layer.

Table S4. The prediction performance of DeephageTP on the seven testing datasets and the mimic dataset (group 8).

Table S5. The prediction performance of DeephageTP with cutoff values on the seven testing datasets and the mimic dataset (group 8).

Table S6. The manual-check result of the protein sequences identified by DeephageTP.

\section{References}

1. Ogilvie LA, Bowler LD, Caplin J, Dedi C, Diston D, Cheek E, Taylor H, Ebdon JE, Jones BV: Genome signature-based dissection of human gut metagenomes to extract subliminal viral sequences. Nature communications 2013, 4(1):1-16.

2. $\quad$ Edwards RA, Rohwer F: Viral metagenomics. Nature Reviews Microbiology 2005, 3(6):504. 
3. Pedulla ML, Ford ME, Houtz JM, Tharun K, Curtis W, Lewis JA, Debbie JS, Jacob F, Joseph G, Pannunzio NR: Origins of highly mosaic mycobacteriophage genomes. Cell 2003, 113(2):171-182.

4. $\quad$ Altschul SF, Madden TL, Schäffer AA, Zhang J, Zhang Z, Miller W, Lipman DJ: Gapped BLAST and PSIBLAST: a new generation of protein database search programs. Nucleic Acids Research 1997, 25:3389-3402.

5. Finn RD, Clements J, Eddy SR: HMMER web server: interactive sequence similarity searching. Nucleic Acids Res 2011, 39(Web Server issue):W29-37.

6. Seguritan V, Alves N, Jr., Arnoult M, Raymond A, Lorimer D, Burgin AB, Jr., Salamon P, Segall AM: Artificial neural networks trained to detect viral and phage structural proteins. PLoS Comput Biol 2012, 8(8):e1002657.

7. Feng PM, Ding H, Chen W, Lin H: Naïve Bayes classifier with feature selection to identify phage virion proteins. Computational and Mathematical Methods in Medicine,2013,(2013-5-14) 2013, 2013(2):530696. Hui D, Peng-Mian F, Wei C, Hao L: Identification of bacteriophage virion proteins by the ANOVA feature selection and analysis. Molecular Biosystems 2014, 10(8):2229-2235.

9. Zhang L, Zhang C, Gao R, Yang R: An Ensemble Method to Distinguish Bacteriophage Virion from Non-

10. Galiez C, Magnan CN, Coste F, Baldi P: VIRALpro: a tool to identify viral capsid and tail sequences.

11. Manavalan B, Shin TH, Lee G: PVP-SVM: Sequence-Based Prediction of Phage Virion Proteins Using a

12. Pan Y, Gao H, Lin H, Liu Z, Tang L, Li S: Identification of Bacteriophage Virion Proteins Using Multinomial

13. Tan J-X, Dao F-Y, Lv H, Feng P-M, Ding H: Identifying phage virion proteins by using two-step feature selection methods. Molecules 2018, 23(8):2000.

15. Li Y, Wang S, Umarov R, Xie B, Fan M, Li L, Gao X: DEEPre: sequence-based enzyme EC number prediction by deep learning. Bioinformatics 2018, 34(5):760-769.

16. Zou Z, Tian S, Gao X, Li YJFig: mldeepre: Multi-functional enzyme function prediction with hierarchical multi-label deep learning. Frontiers in genetics 2018, 9:714.

17. Zhang F, Song H, Zeng M, Li Y, Kurgan L, Li MJP: DeepFunc: A Deep Learning Framework for Accurate Prediction of Protein Functions from Protein Sequences and Interactions. Proteomics 2019:1900019.

18. Kulmanov M, Khan MA, Hoehndorf R: DeepGO: predicting protein functions from sequence and interactions using a deep ontology-aware classifier. Bioinformatics 2017, 34(4):660-668.

19. Abid D, Zhang LJb: DeepCapTail: A Deep Learning Framework to Predict Capsid and Tail Proteins of Phage Genomes. bioRxiv 2018:477885.

20. Gao S, Zhang L, Rao VB: Exclusion of small terminase mediated DNA threading models for genome packaging in bacteriophage T4. Nucleic Acids Res 2016, 44(9):4425-4439.

21. Hilbert BJ, Hayes JA, Stone NP, Xu RG, Kelch BA: The large terminase DNA packaging motor grips DNA with its ATPase domain for cleavage by the flexible nuclease domain. Nucleic Acids Res 2017, 45(6):35913605.

22. Moreno-Gallego JL, Chou S-P, Di Rienzi SC, Goodrich JK, Spector TD, Bell JT, Youngblut ND, Hewson I, Reyes A, Ley REJCh et al: Virome diversity correlates with intestinal microbiome diversity in adult monozygotic twins. Cell host \& microbe 2019, 25(2):261-272. e265. 
23. Yinda CK, Vanhulle E, Conceição-Neto N, Beller L, Deboutte W, Shi C, Ghogomu SM, Maes P, Van Ranst M, Matthijnssens JJm: Gut Virome Analysis of Cameroonians Reveals High Diversity of Enteric Viruses, Including Potential Interspecies Transmitted Viruses. mSphere 2019, 4(1):e00585-00518.

24. Bankevich A, Nurk S, Antipov D, Gurevich AA, Dvorkin M, Kulikov AS, Lesin VM, Nikolenko SI, Pham S, Prjibelski ADJJocb: SPAdes: a new genome assembly algorithm and its applications to single-cell sequencing. Journal of computational biology 2012, 19(5):455-477.

25. Hyatt D, Chen G-L, LoCascio PF, Land ML, Larimer FW, Hauser LJJBb: Prodigal: prokaryotic gene recognition and translation initiation site identification. BMC bioinformatics 2010, 11(1):119.

26. LeCun Y, Bengio Y, Hinton G: Deep learning Nature 2015, 521(7553):436.

27. Srivastava N, Hinton G, Krizhevsky A, Sutskever I, Salakhutdinov RJTjomlr: Dropout: a simple way to prevent neural networks from overfitting. The journal of machine learning research 2014, 15(1):1929-1958.

28. Zang F, Zhang J-s: Softmax discriminant classifier. In: 2011 Third International Conference on Multimedia Information Networking and Security: 2011. IEEE: 16-19.

29. Zeng H, Edwards MD, Liu G, Gifford DKJB: Convolutional neural network architectures for predicting DNA-protein binding. 2016, 32(12):i121-i127.

30. Arango-Argoty G, Garner E, Pruden A, Heath LS, Vikesland P, Zhang L: DeepARG: a deep learning approach for predicting antibiotic resistance genes from metagenomic data. Microbiome 2018, 6(1):23.

31. Savojardo C, Martelli PL, Fariselli P, Casadio RJB: DeepSig: deep learning improves signal peptide detection in proteins. 2017, 34(10):1690-1696.

32. Suresh V, Liu L, Adjeroh D, Zhou XJNar: RPI-Pred: predicting ncRNA-protein interaction using sequence and structural information. 2015, 43(3):1370-1379.

33. Yi H-C, You Z-H, Zhou X, Cheng L, Li X, Jiang T-H, Chen Z-HJMT-NA: ACP-DL: A Deep Learning Long Short-Term Memory Model to Predict Anticancer Peptides Using High-Efficiency Feature Representation. 2019, 17:1-9.

34. Eddy SRJPcb: Accelerated profile HMM searches. PLoS computational biology 2011, 7(10):e1002195.

35. Edgar RCJNar: MUSCLE: multiple sequence alignment with high accuracy and high throughput. Nucleic Acids Research 2004, 32(5):1792-1797.

36. Buchfink B, Xie C, Huson DHJNm: Fast and sensitive protein alignment using DIAMOND. Nature methods 2015, 12(1):59. 
621 Tables

622 Table 1. The numbers of proteins of each category in the training dataset.

\begin{tabular}{ccc}
\hline Protein categories & \multicolumn{2}{c}{ Training dataset } \\
\cline { 2 - 3 } & $80 \%$ train-set & $20 \%$ test-set \\
\hline \# TerL & 2093 & 524 \\
\# Portal & 2607 & 653 \\
\# TerS & 1202 & 301 \\
\# others & 16163 & 4042 \\
\hline
\end{tabular}

$62380 \%$ train-set and 20\% test-set are used for feasibility analysis, and the training dataset (including 624 train-set and test-set) is used for training the proposed model.

625

626

627

628

629

630

631

632

633

634

635

636

637

638

639

640

641

642

643

644 
Table 2. The numbers of proteins of each category in the mimic metagenomic dataset and the seven testing groups.

\begin{tabular}{ccccc}
\hline $\begin{array}{c}\text { Testing } \\
\text { datasets }\end{array}$ & \# TerL & \# Portal & \# TerS & \# Others \\
\hline Group 1 & 14437 & 41398 & 5918 & 30000 \\
\hline Group 2 & 14437 & 41398 & 5918 & 50000 \\
\hline Group 3 & 14437 & 41398 & 5918 & 70000 \\
\hline Group 4 & 14437 & 41398 & 5918 & 90000 \\
\hline Group 5 & 14437 & 41398 & 5918 & 110000 \\
\hline Group 6 & 14437 & 41398 & 5918 & 130000 \\
\hline Group 7 & 14437 & 41398 & 5918 & 150000 \\
\hline Group 8 & 14437 & 41398 & 5918 & 476685 \\
\hline
\end{tabular}

647 
Table 3. Comparison of prediction performances of the multiclass classification model and binary classification model on the test-set of the training dataset.

\begin{tabular}{ccccc|cccc}
\hline \multirow{2}{*}{ Proteins } & \multicolumn{3}{c|}{ Multiclass classification } & \multicolumn{3}{c}{ Binary classification } \\
\cline { 2 - 9 } & Accuracy & Precision & Recall & F1 & Accuracy & Precision & Recall & F1 \\
\hline TerL & 0.9858 & 0.9375 & 0.916 & 0.9267 & $\mathbf{0 . 9 8 8 2}$ & $\mathbf{0 . 9 5 4 5}$ & $\mathbf{0 . 9 1 9 8}$ & $\mathbf{0 . 9 3 6 8}$ \\
\hline Portal & 0.9884 & 0.9378 & $\mathbf{0 . 9 6 9 4}$ & 0.9533 & $\mathbf{0 . 9 9 2 4}$ & $\mathbf{0 . 9 9 1 9}$ & 0.9433 & $\mathbf{0 . 9 6 7}$ \\
\hline TerS & $\mathbf{0 . 9 7 8 3}$ & $\mathbf{0 . 7 5 2 8}$ & 0.9103 & $\mathbf{0 . 8 2 4 1}$ & 0.9696 & 0.658 & 0.9203 & 0.7673 \\
\hline
\end{tabular}


Figure Legends

Fig. 1. Overview of the framework DeephageTP. (A) The workflow of the proposed DeephageTP framework. The CNN-based model was firstly implemented on the training dataset. And then the trained model was applied on the mimic metagenomic dataset and the cutoff loss value of each category of interest was determined. Finally, the trained model was applied to the real metagenomic datasets for validating the performance of our framework. (B) One-hot encoding for protein sequence. Each amino acid is represented as a one-hot vector. (C) The process of the CNN-based model. The final classification is performed by a standard fully-connected neural network.

Fig. 2. Prediction performance of the CNN-based model. (A) Performance of the model on the training data. The model was trained on the train-set ( $80 \%$ training data), and the prediction performance was evaluated on the test-set (20\% training data) with four metrics (i.e., Accuracy, Precision, Recall and, F1-score) for the three phage proteins, respectively. (B) Comparison of the prediction performance of the model on the test-set of the training dataset and the mimic metagenomic dataset. The prediction performances for two datasets (purple: the test-set of the training dataset, green: the mimic dataset) were evaluated with four metrics (i.e., Accuracy, Precision, Recall and, F1-score) for the three phage proteins, respectively.

Fig. 3. Performances of the model with and without cutoff loss values on the mimic metagennomics dataset. The performance was evaluated in terms of Precision (Precision 1, without cutoff loss values; Precision 2, with cutoff loss values). 7 groups (Group 1 7) with different sizes were generated from the mimic metagenomic dataset.

Fig. 4. The loss value distributions of TP and FP for the three phage proteins on the mimic metagenomic dataset. Group 1 7 datasets were generated from the mimic metagenomic dataset (group 8). The loss value distributions of TP (all are the same for eight groups) and FP were calculated on the eight groups, respectively, for the three phage proteins. TP: true positive; FP: false positive. g1 g8: Group1 Group8.

Fig. 5. Venn diagrams of the prediction results of three methods (i.e., DeephageTP, Diamond and HMMER) on the metagenomic dataset (SRR5192446). A: TerL; B: Portal; C: TerS.

Fig. 6. Verification of the three phage proteins identified by DeephageTP from the real metagenome datasets. Our method was applied to the three real metagenomic datasets (Sample1: SRR5192446, Sample2: SRR7892426 and Sample3: ERR2868024) for identifying the three phage proteins and the results were verified by Blastp against NCBI nr database. a) true positive: the 
sequence has Blastp hits in the NCBI nr database within the same category as DeephageTP predicted

736 (as long as one hit in the result list of Blastp against NCBI nr database is annotated to the category of interest); b) phage-related: at least one of the protein sequences carried by the contig where the identified protein gene is located has hits to other phage-related proteins (as long as one is annotated to phage-related protein in the result list of Blastp); c) Unknown, the sequences don't have any hits or the hits are annotated as hypothetical protein; d) Other functional, the sequences have hits annotated as other functional proteins that likely are derived from bacterial genomes (none of the hits in the result list of Blastp are annotated as phage-related proteins). 
A.

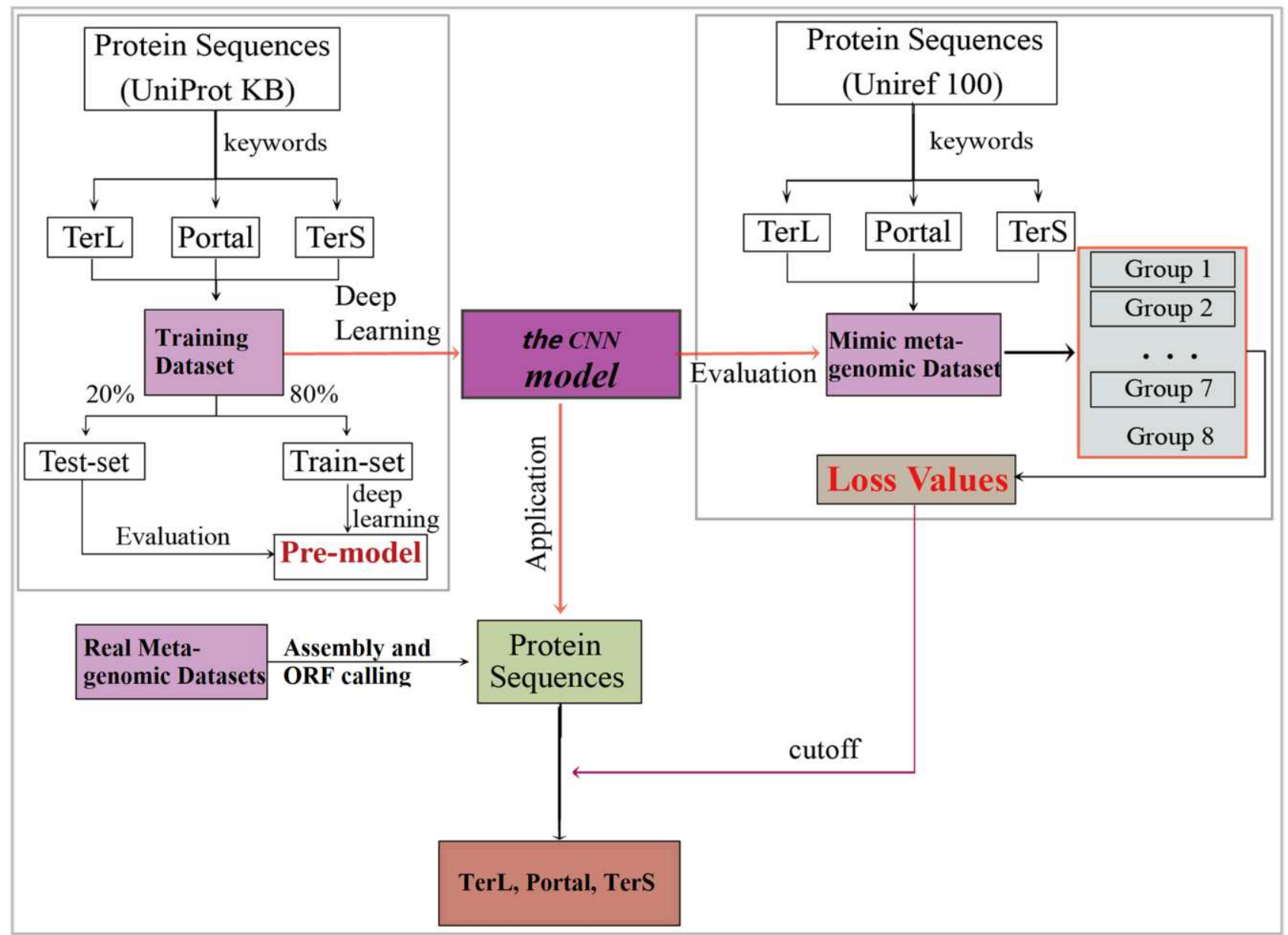

B.

C. Input Layer Feature

Output
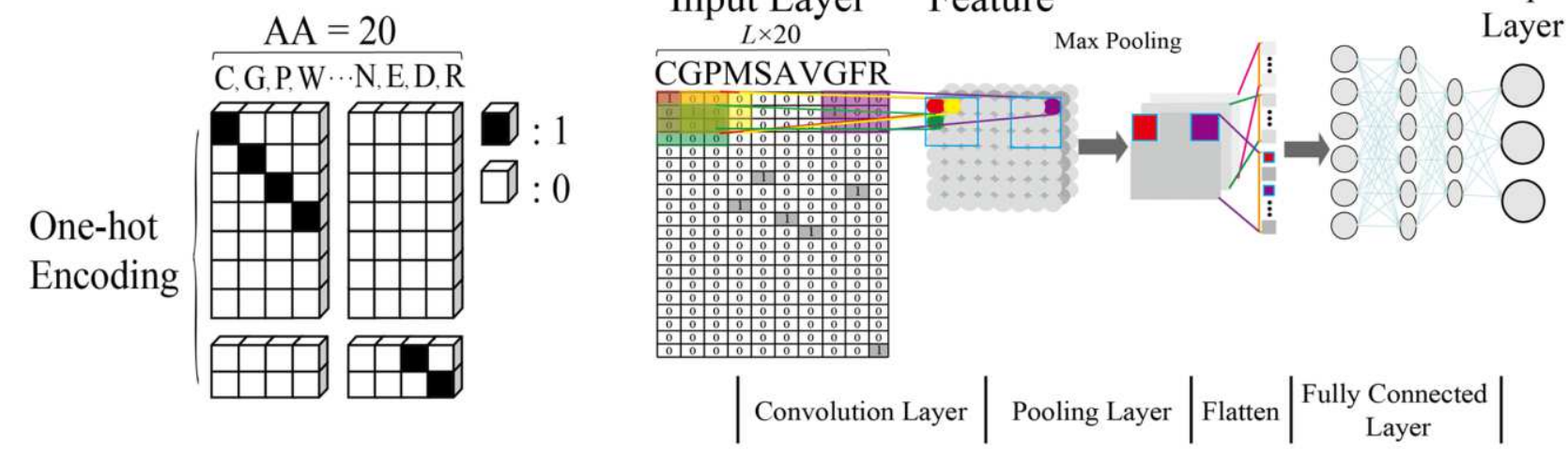
Fig. 2

A.

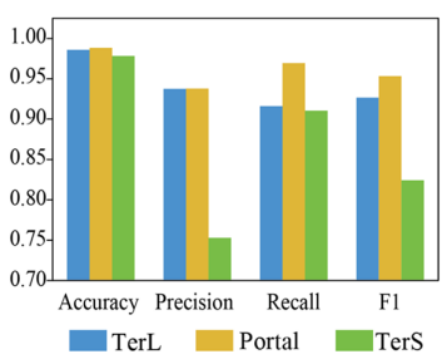

B.

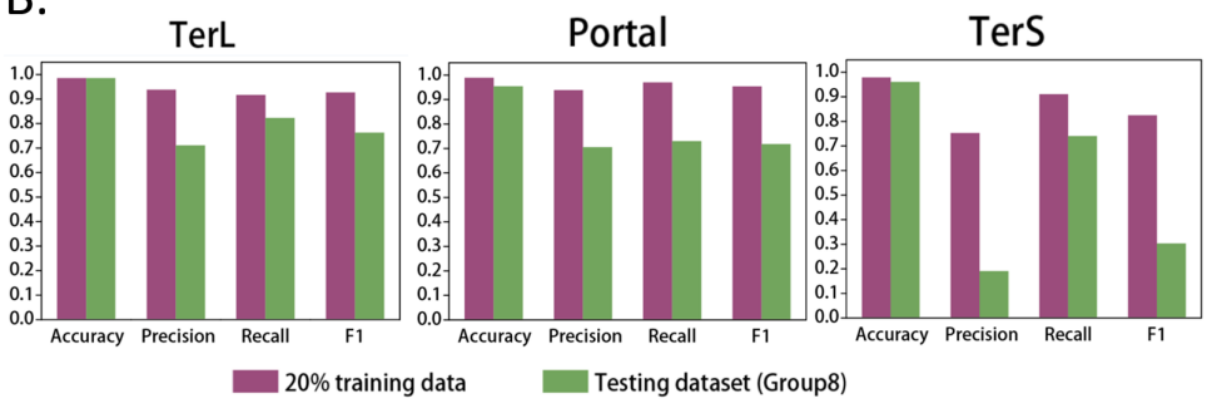


Fig. 3

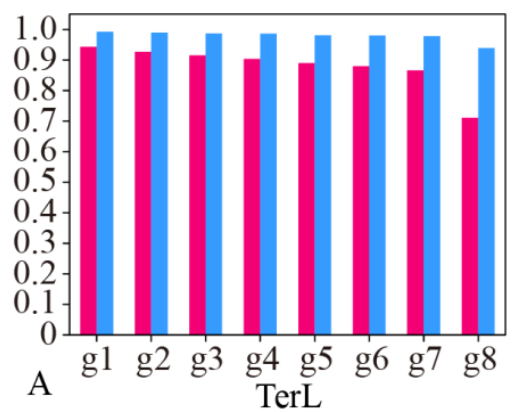

801

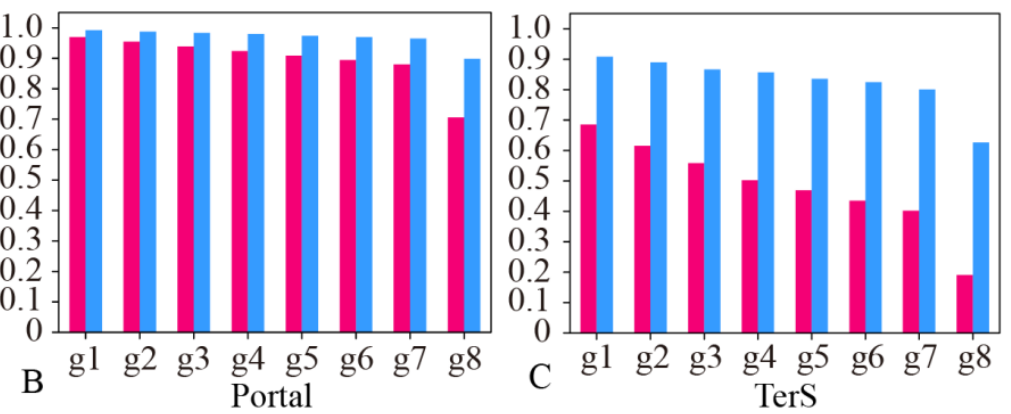

Precision 1

Precision 2

802

803

804

805

806

807

808

809

810

811

812

813

814

815

816

817

818

819

820

821

822

823

824

825 
Fig. 4

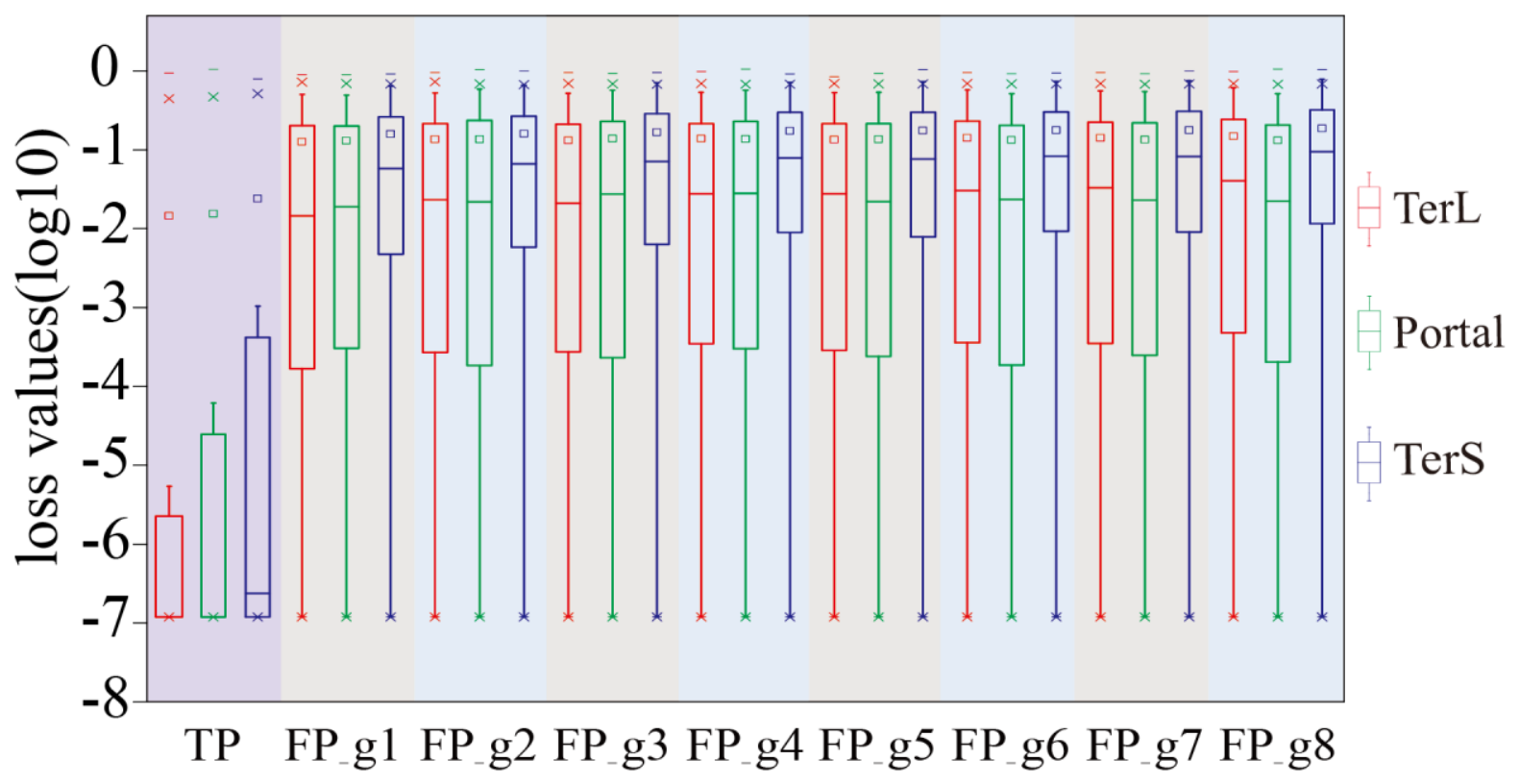

827

828

829

830

831

832

833

834

835

836

837

838

839

840

841

842

843

844

845

846 
Fig. 5
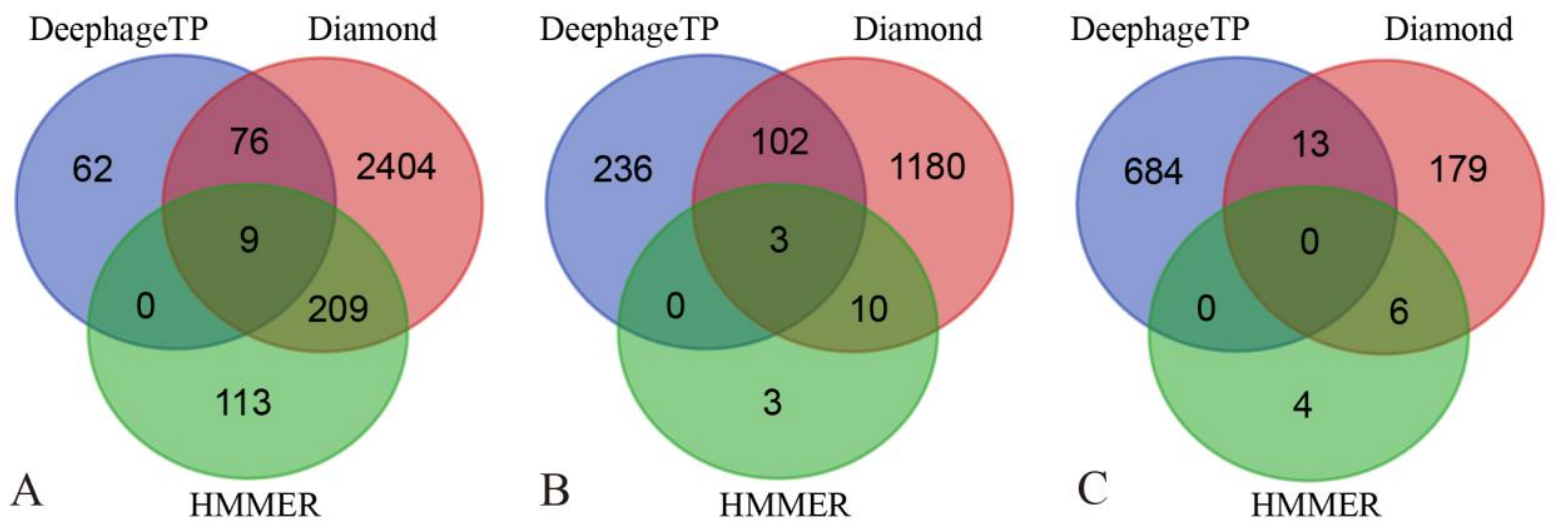

849

850

851

852

853

854

855

856

857

858

859

860

861

862

863

864

865

866

867

868

869

870

871 
Fig. 6

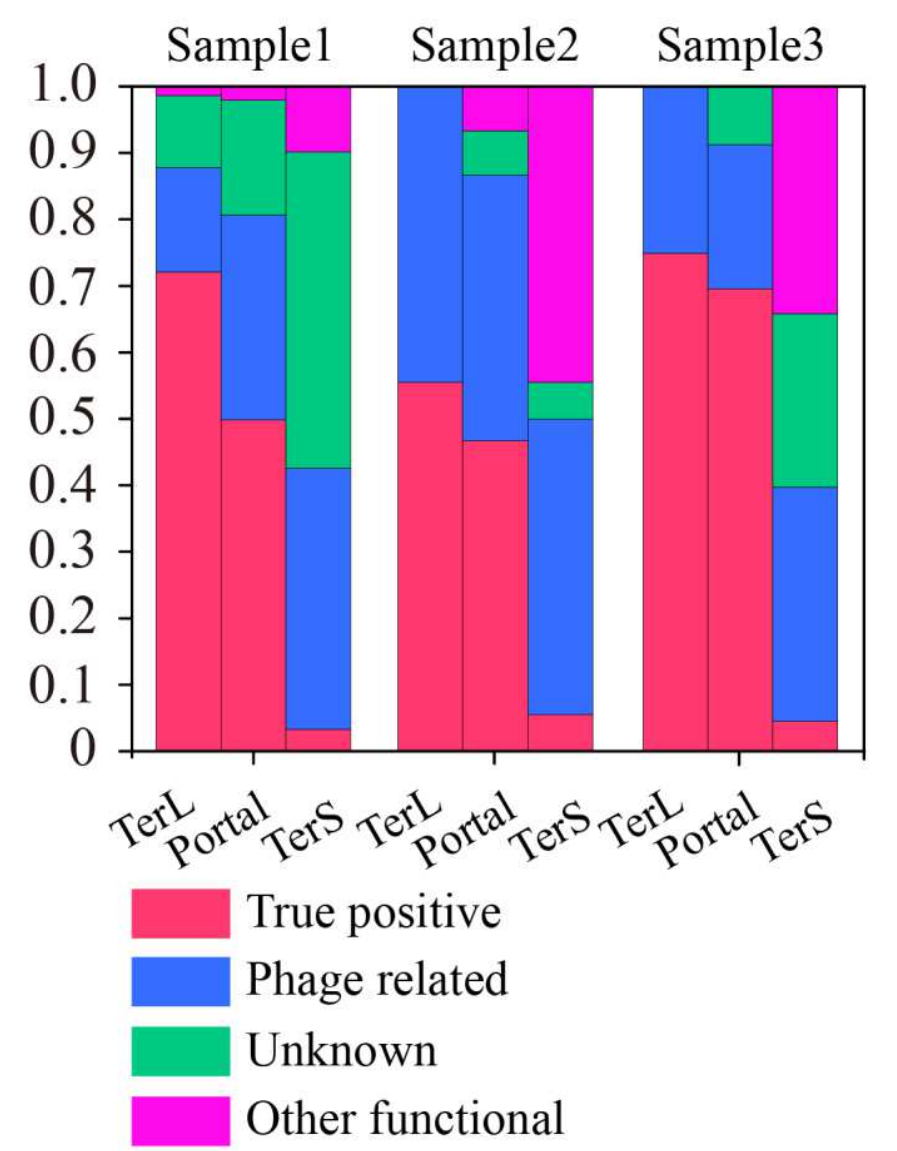




\section{Figures}

A.

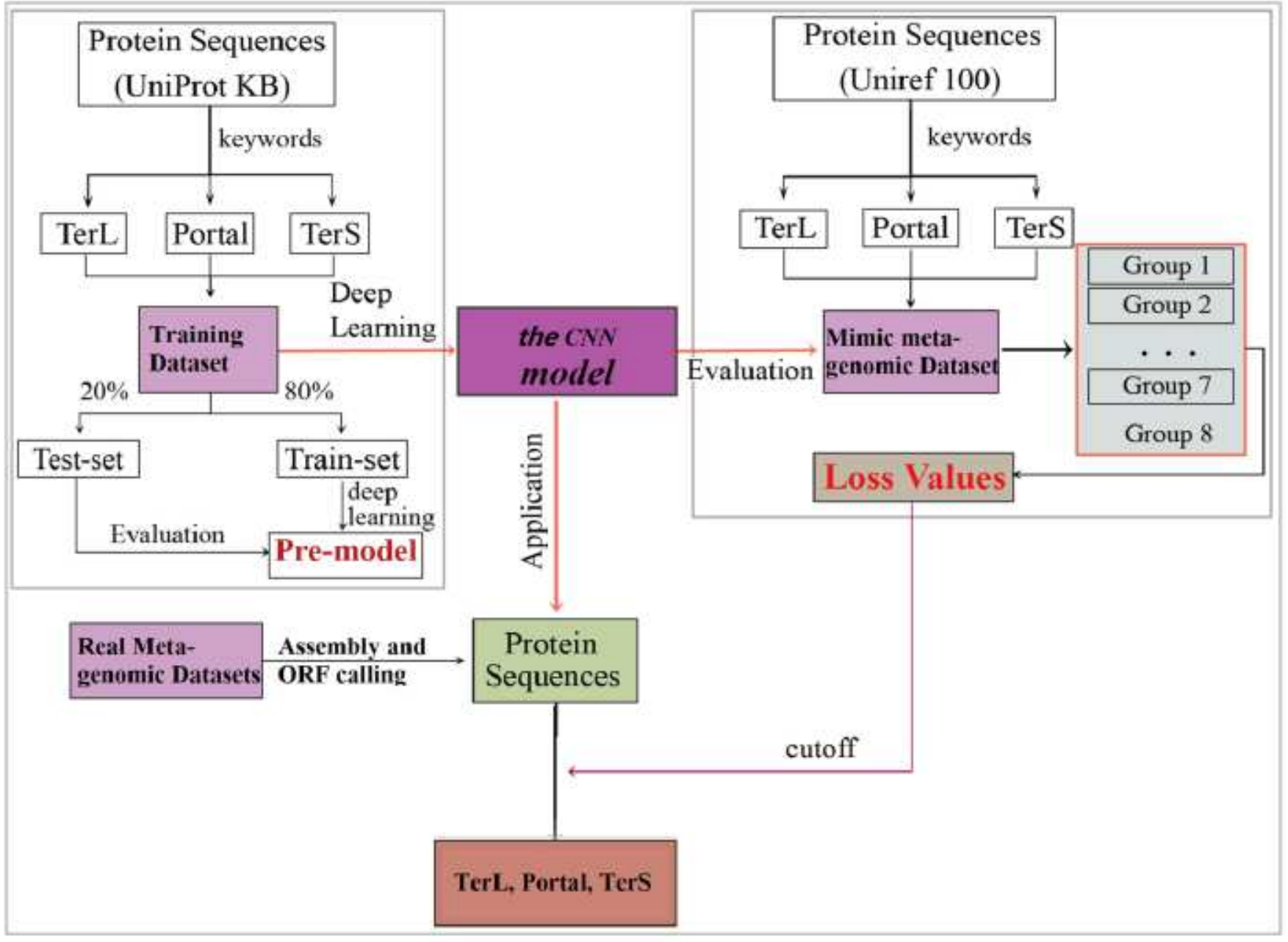

B.

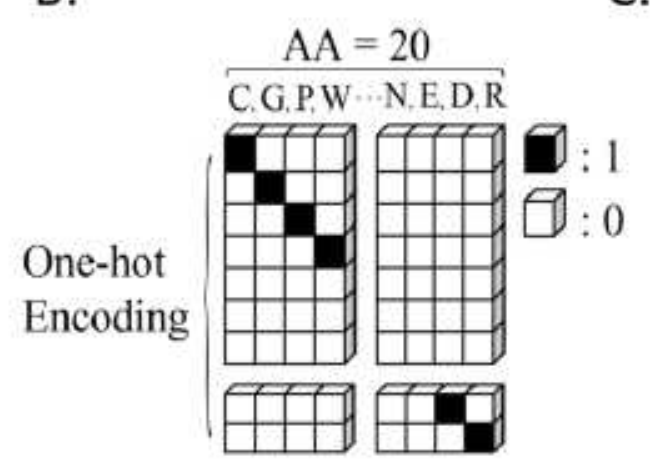

C. Input Layer Fcature

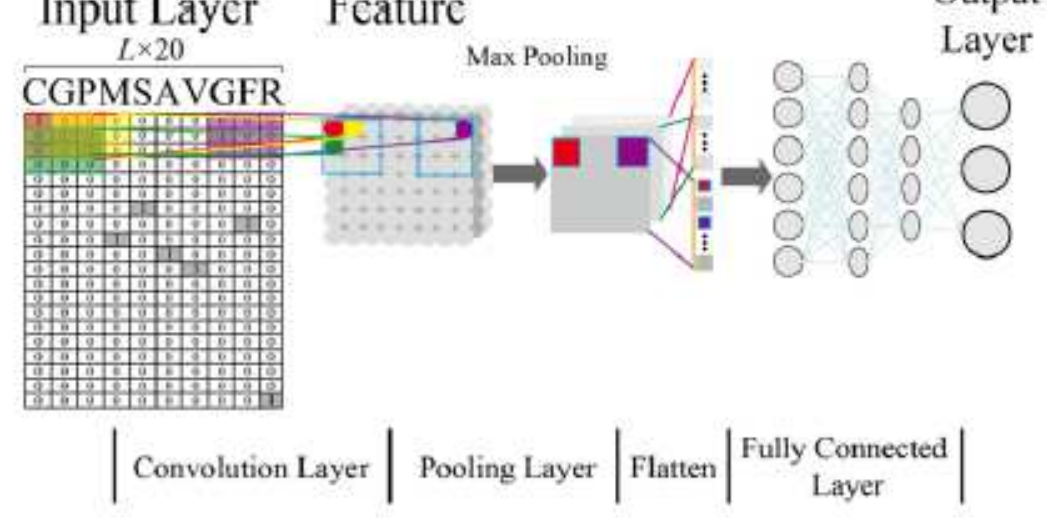

Figure 1

Overview of the framework DeephageTP. (A) The workflow of the proposed DeephageTP framework. The CNN-based model was firstly implemented on the training dataset. And then the trained model was applied on the mimic metagenomic dataset and the cutoff loss value of each category of interest was 
determined. Finally, the trained model was applied to the real metagenomic datasets for validating the performance of our framework. (B) One-hot encoding for protein sequence. Each amino acid is represented as a one-hot vector. (C) The process of the CNN-based model. The final classification is performed by a standard fully-connected neural network.

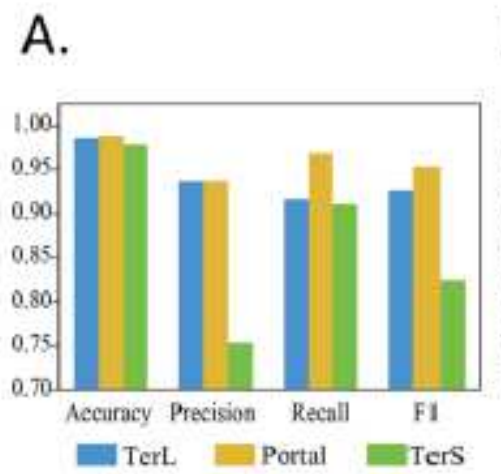

B.
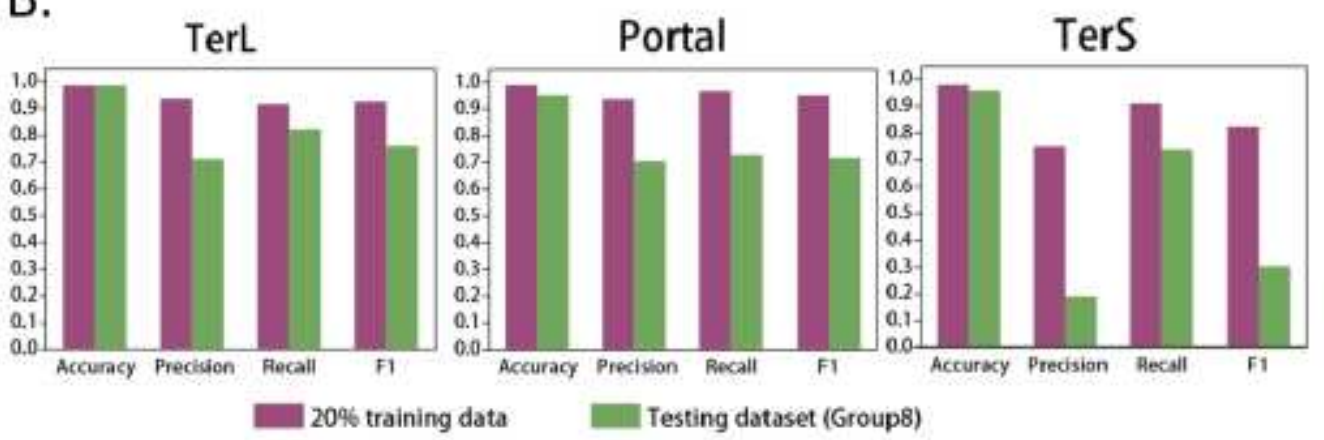

Figure 2

Prediction performance of the CNN-based model. (A) Performance of the model on the training data. The model was trained on the train-set ( $80 \%$ training data), and the prediction performance was evaluated on the test-set (20\% training data) with four metrics (i.e., Accuracy, Precision, Recall and, F1-score) for the three phage proteins, respectively. (B) Comparison of the prediction performance of the model on the testset of the training dataset and the mimic metagenomic dataset. The prediction performances for two datasets (purple: the test-set of the training dataset, green: the mimic dataset) were evaluated with four metrics (i.e., Accuracy, Precision, Recall and, F1-score) for the three phage proteins, respectively.

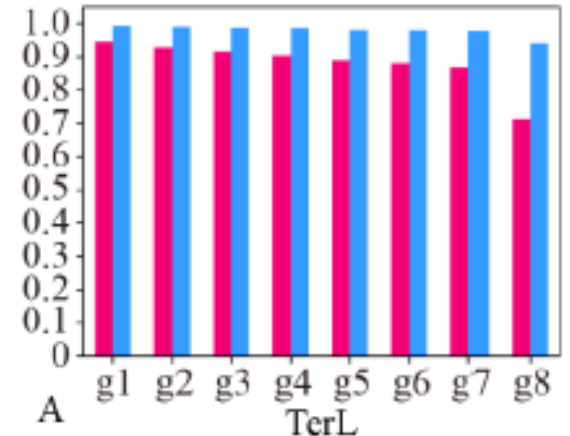

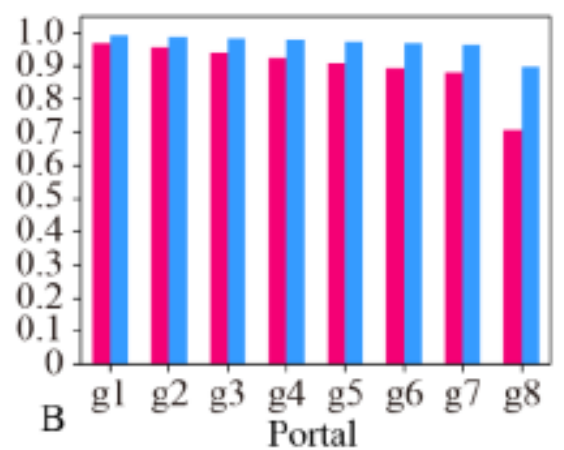

Portal

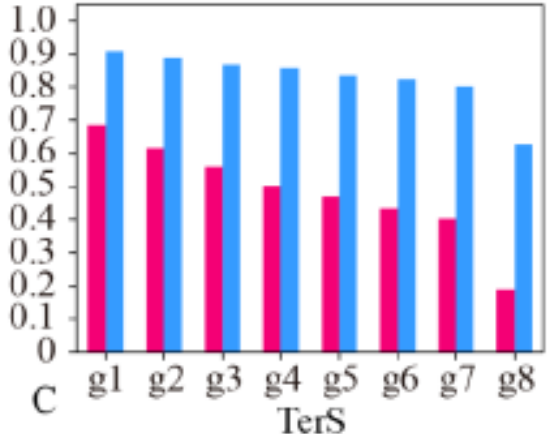

Precision 2

\section{Figure 3}

Performances of the model with and without cutoff loss values on the mimic metagennomics dataset.

The performance was evaluated in terms of Precision (Precision 1, without cutoff loss values; Precision 2, with cutoff loss values). 7 groups (Group 1 7) with different sizes were generated from the mimic metagenomic dataset. 


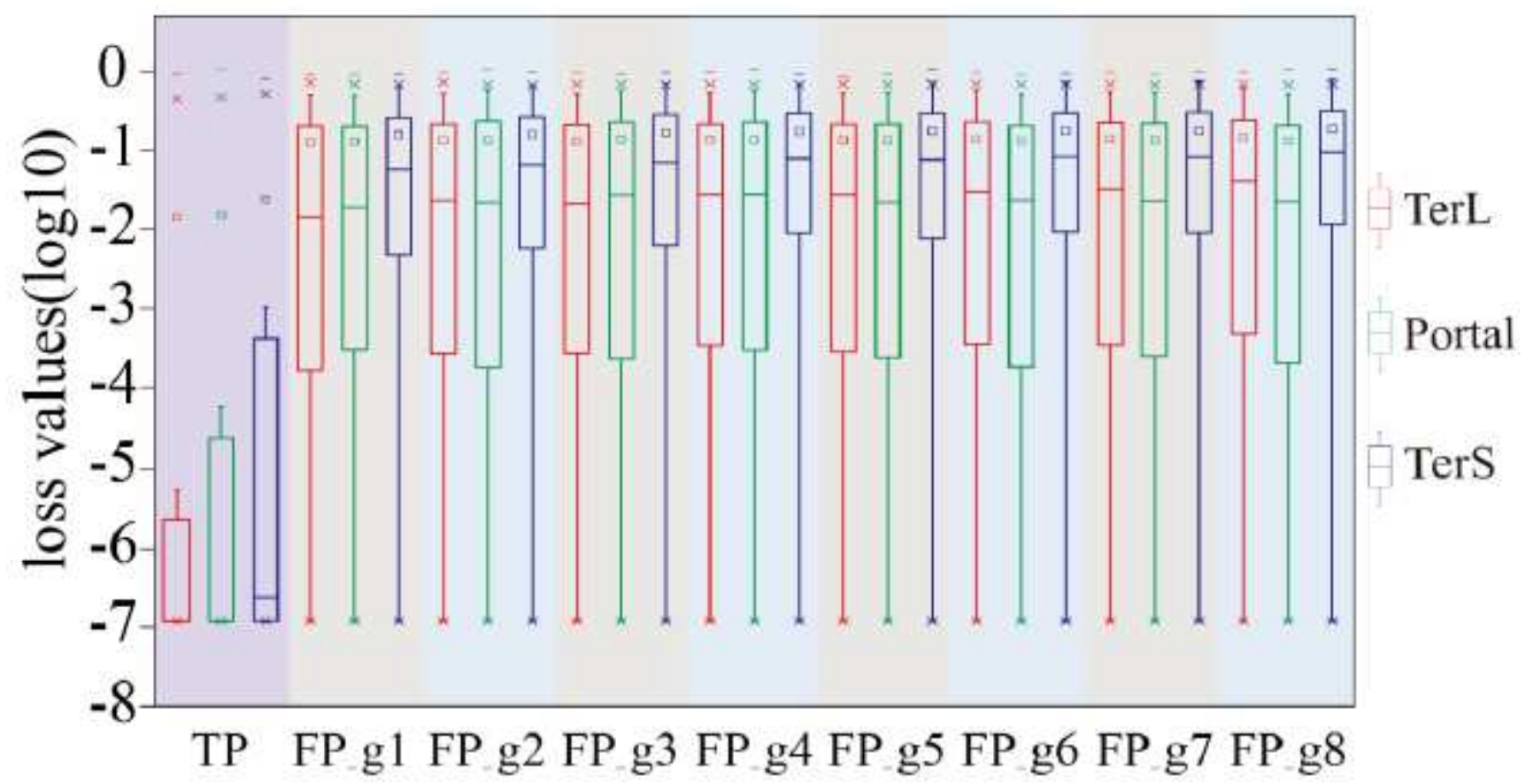

Figure 4

The loss value distributions of TP and FP for the three phage proteins on the mimic metagenomic dataset. Group 1 7 datasets were generated from the mimic metagenomic dataset (group 8). The loss value distributions of TP (all are the same for eight groups) and FP were calculated on the eight groups, respectively, for the three phage proteins. TP: true positive; FP: false positive. g1 g8: Group1 Group8.

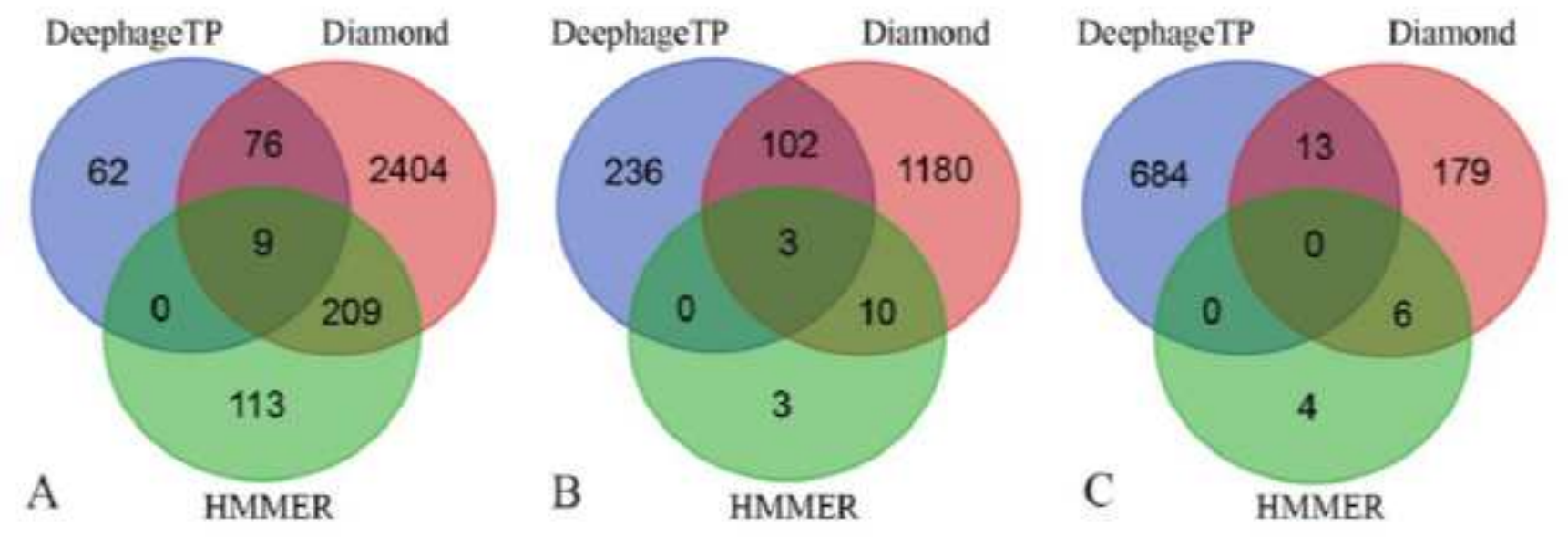

Figure 5

Venn diagrams of the prediction results of three methods (i.e., DeephageTP, Diamond and HMMER) on the metagenomic dataset (SRR5192446). A: TerL; B: Portal; C: TerS. 


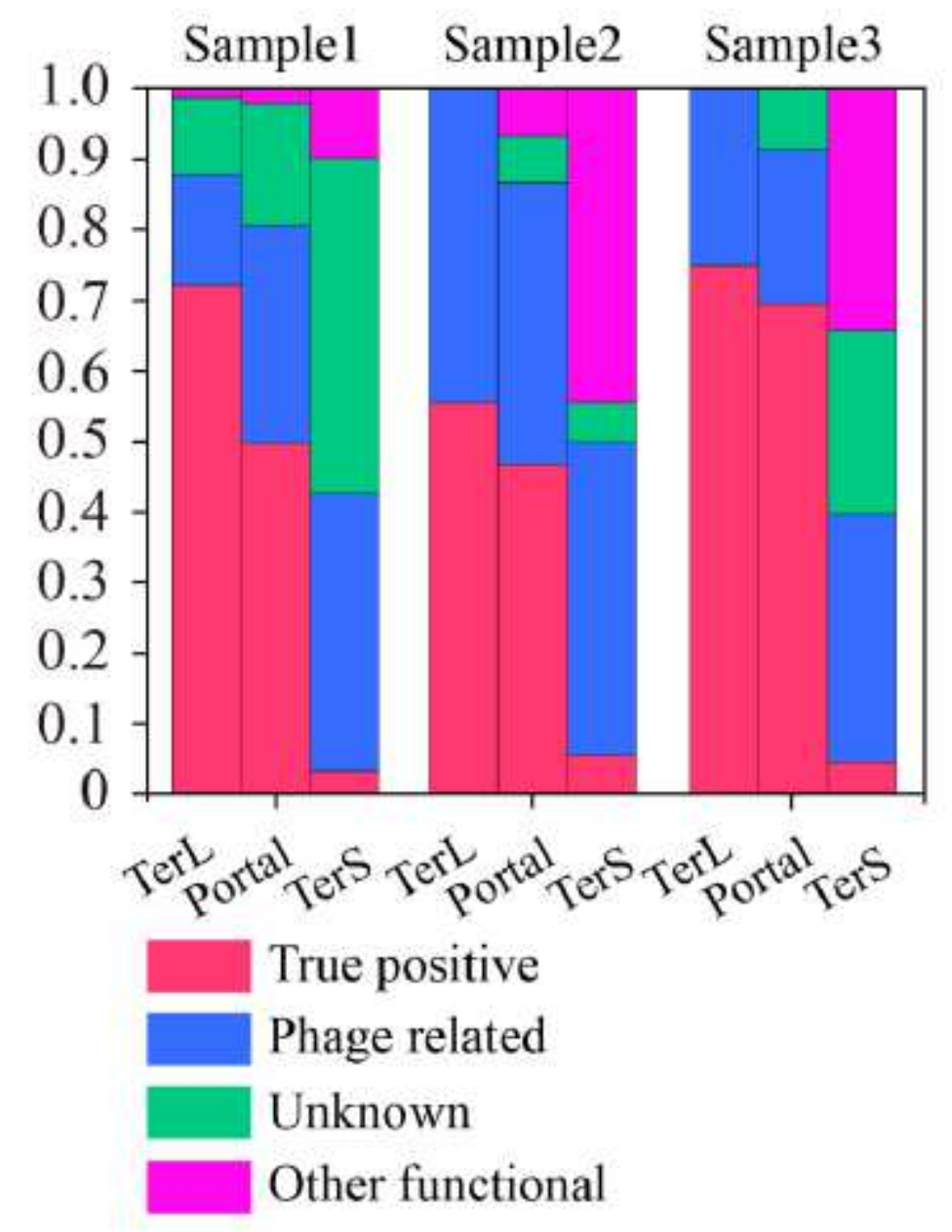

\section{Figure 6}

Verification of the three phage proteins identified by DeephageTP from the real 731 metagenome datasets. Our method was applied to the three real metagenomic datasets (Sample1: 732 SRR5192446, Sample2: SRR7892426 and Sample3: ERR2868024) for identifying the three phage proteins and the results were verified by Blastp against NCBI nr database. a) true positive: the sequence has Blastp hits in the NCBI nr database within the same category as DeephageTP predicted (as long as one hit in the result list of Blastp against NCBI nr database is annotated to the category of interest); b) phage-related: at least one of the protein sequences carried by the contig where the identified protein gene is located has hits to other phage-related proteins (as long as one is annotated to phage-related protein in the result list of Blastp); c) Unknown, the sequences don't have any hits or the hits are annotated as hypothetical protein; d) Other functional, the sequences have hits annotated as other functional proteins that likely are derived from bacterial genomes (none of the hits in the result list of Blastp are annotated as phage-related proteins).

\section{Supplementary Files}


This is a list of supplementary files associated with this preprint. Click to download.

- SupplementaryTablesandFigures.xls 